\title{
Chest image staging and prevalence of current smoking among hospitalized COVID-19 patients in Turkey
}

\author{
Sinan Eroğlu ${ }^{1}$, Eren Şahin ${ }^{2}$, Şule Yoluç ${ }^{2}$, Yasemin Eroğlu ${ }^{3}$, and İmran Aydoğdu² \\ ${ }^{1}$ Istanbul Bahçelievler State Hospital \\ ${ }^{2}$ Istanbul Bahcelievler State Hospital \\ ${ }^{3}$ Istanbul Bağcılar Eğitim ve Araştırma Hastanesi
}

November 28, 2020

\begin{abstract}
Objective: Novel Coronavirus disease is a new infectious agent of the respiratory tract characterized by a severe acute respiratory syndrome. For this disease, there are limited data with regard to the clinical characteristics of the patients and prognostic factors. Study Design: Retrospective Cohort Setting: Secondary Referral Center Methods: We collected data from 213 patients who were hospitalized into COVID-19 isolation with positive PCR test results. We recorded various patient values, including blood test results. We also noted age, gender, additional diseases, duration of discharge, whether they live or die, whether they smoke, and their radiological staging. Results: In CT imaging with a staging of maximum 4 points and minimum 0 points, the mean value resulted in 1.95. The average radiological stage of the dead patients group was reported as 2.56. There was a correlation between the radiological predictor and the outcome status (p-value: 0.002 ). The number of smokers was 14 (6.5\%). Of the 26 patients who died, 3 were smokers and 23 were non-smokers. Conclusion: 14 of the patients in the study were smokers (6.5\%). One in four people in Turkey is a smoker, while in COVID-19 isolation service only a $6.5 \%$ rate of smoking was observed. That supports the theory that smoking hasn't negative impact on COVID-19 development. The average radiological stage was reported as 2.56 in the dead patients's group. There was a correlation between the radiological predictor and the outcome status (p-value: 0.002). It seems that an elevated radiological stage is a predictor of death. Keywords: Covid-19, SARS-CoV-2, smoking, computed tomography, predictor factors. Key points: to learn relations between smoking and covid-19, effect of Ct stages on the disease severity, effect of blood analysis on Covid-19, the parameters in deaths of Covid-19, ratio of smoking in Covid-19 inpatients
\end{abstract}

\section{Chest image staging and prevalence of current smoking among hospitalized COVID-19 patients in Turkey}

\section{Abstact:}

Objective: Novel Coronavirus disease is a new infectious agent of the respiratory tract characterized by a severe acute respiratory syndrome. For this disease, there are limited data with regard to the clinical characteristics of the patients and prognostic factors.

Study Design: Retrospective Cohort

\section{Setting: Secondary Referral Center}

Methods: We collected data from 213 patients who were hospitalized into COVID-19 isolation with positive PCR test results. We recorded various patient values, including blood test results. We also noted age, gender, additional diseases, duration of discharge, whether they live or die, whether they smoke, and their radiological staging. 
Results: In CT imaging with a staging of maximum 4 points and minimum 0 points, the mean value resulted in 1.95. The average radiological stage of the dead patients group was reported as 2.56. There was a correlation between the radiological predictor and the outcome status (p-value: 0.002). The number of smokers was $14(6.5 \%)$. Of the 26 patients who died, 3 were smokers and 23 were non-smokers.

Conclusion: 14 of the patients in the study were smokers (6.5\%). One in four people in Turkey is a smoker, while in COVID-19 isolation service only a $6.5 \%$ rate of smoking was observed. That supports the theory that smoking hasn't negative impact on COVID-19 development. The average radiological stage was reported as 2.56 in the dead patients's group. There was a correlation between the radiological predictor and the outcome status (p-value: 0.002). It seems that an elevated radiological stage is a predictor of death.

Keywords: Covid-19, SARS-CoV-2, smoking, computed tomography, predictor factors.

Key points : to learn relations between smoking and covid-19, effect of Ct stages on the disease severity, effect of blood analysis on Covid-19, the parameters in deaths of Covid-19, ratio of smoking in Covid-19 inpatients Chest image staging and prevalence of current smoking among hospitalized COVID-19 patients
in Turkey

\section{Introduction:}

Novel Coronavirus disease (COVID-19) is a new infectious agent of the respiratory tract characterized by a severe acute respiratory syndrome (SARS). SARS-CoV-2 started in Wuhan, China in December 2019 and quickly evolved into a rapidly spreading pandemic ${ }^{1}$. Because of its high infectivity and mortality, it is a serious public health threat ${ }^{2}$.

Although most patients are asymptomatic or have mild symptoms and a good prognosis, COVID-19 can progress to more severe illnesses, including pneumonia, acute respiratory distress syndrome, multiple organ failure, or even death ${ }^{3,4}$. The aim of successful treatment is reduction of complications and mortality. Basic disease treatment is also needed to prevent secondary infection.

For this disease, there are limited data with regard to the clinical characteristics of the patients and prognostic factors $^{5}$. Chronic diseases and old age have been assumed to be associated with adverse disease prognosis. Little attention has been given to the role of smoking in the transmission of SARS-CoV2. Smokers contract more respiratory infections including colds (commonly rhinoviruses), than non-smokers. Smokers also develop influenza twice as often and show increased rates of bacterial pneumonia and tuberculosis. The damage caused to the lungs by smoking makes these patients more susceptible to pulmonary infections, both bacterial and viral ${ }^{6}$.

There are two opposing opinions about the effect of smoking on COVID-19's severity. It has recently been reported that ACE2 gene expression, which is known to be a cellular entry gateway for SARS-CoV-2, is higher in smokers. This expression is also the upregulation associated with smoking, this suggests that smoking contributes to a higher number of viral receptors and may support the findings of the recent case series $\operatorname{research}^{7,8}$. However, the second opinion is that smoking prevents downregulation of angiotensin-converting enzyme 2 (ACE2) and that this downregulation is harmful due to uncontrolled ACE2 and angiotensin II activity $^{9}$. It has been observed that decreased ACE2 availability contributes to lung injury and increases the chance of development of acute respiratory distress syndrome (ARDS) ${ }^{9}$.

Therefore, it is very important to find the related factors of disease severity in clinical practice. In this study, we compared the clinic and laboratory findings and computed tomography (CT) features of 223 ordinary COVID-19 cases.

\section{Method:}

This study was done based on the data of the patients hospitalized in the COVID-19 isolation department in Istanbul Bahçelievler State Hospital. 
It was performed on 223 patients who were evaluated in the emergency department and then hospitalized between 11 April and 17 May 2020 and whose COVID-19 polymerase chain reaction (PCR) test result was positive. Within the scope of the COVID-19 protocol we conduct in our hospital, patients with cough, sore throat, and respiratory distress are transferred from the emergency triage to the isolation area. Dependent upon the patient's history, which is taken while a PCR test is administered, hemogram, biochemistry blood tests, and pulmonary computed tomography may be requested. The patient's history includes questions regarding additional illness and smoking. If the patient has respiratory distress, high fever, bad general condition, accompanying chronic disease, old age, and high computed tomography staging we prepare by referring to other studies ${ }^{4,10}$, interneed to the isolated service for COVID-19. According to the directive from the Turkish Ministry of Health, we start our treatment with Plaquenil, Azyhtromycin, Oseltamavir, Clexane 0,6, and a high dose Vitamin C. If oxygen saturations are below 90, Favirapir treatment is initiated. On the first day of the patient's hospitalization, we send routine blood tests for hemogram, full biochemistry, serology, coagulation markers, Troponin, Ferrritin, and D-Dimer levels. If patients have normalized laboratory tests, no fever and no respiratory distress for at least 3 days, and a good general condition, they are discharged with an explanation of isolation rules.

We collected data from 213 patients who were admitted into COVID-19 isolation with positive PCR test results. We recorded various patient values, including white blood cell (Wbc), neutrophil, lymphocyte, platelet, mean platelet volume (MPV), ferritin, D-dimer, troponin-I, c-reactive protein (CRP), magnesium, calcium, creatinine, lactate dehydrogenase (LDH). We also noted age, gender, additional diseases, duration of discharge, whether they live or die, whether they smoke, and their radiological staging.

While inquiring about additional diseases, we use the E-Nabız System that was organized by the Turkish Ministry of Health. This system incorporates a large amount of information, such as what diagnoses patients have had before, the operations they have undergone, and the medications they have been prescribed. For patients who are referred with a pre-diagnosis of COVID-19, smoking-related information is gathered and recorded before the PCR test and confirmed during hospitalization.

In the case of our hospital, chest images were examined by the same radiologist who conducted the earlier tests. A semi-quantitative scoring obtained from CT images was used to determine the severity of COVID19 's pulmonary involvement. Each lung lobe was scored as $0(0 \%), 1(1-25 \%), 2(26-50 \%), 3(51-75 \%)$, and $4(76-100 \%)$ according to the COVID-19 involvement percentage. The total involvement score (0-20) of all 5 lobes was obtained, and CT evaluation was performed in the lung parenchyma window. A Toshiba Alexion device with 16 detectors, extant in our own hospital, was used. Scoring was performed according to the ground glass opacity appearance of COVID-19, which is the typical lung involvement, and the percentage of consolidations. Of the original 223 patients, those with atypical COVID-19 findings $(\mathrm{n}=4)$, those with lung malignancy $(\mathrm{n}=2)$ and those with atelectasis $(\mathrm{n}=4)$ were excluded from the study, leaving 213 participants.

\section{Results:}

Pearson's correlation coefficient and two-sample t-tests were used to evaluate the 213 patients who had a positive COVID-19 PCR test and were hospitalized in the isolation service of our hospital.

Of the 213,118 were male (55.3\%). The average age was 56 years. The number of patients who died was 26 $(12.2 \%)$. The average age of the 26 patients who died was 60.9 years and the majority were men (17 males, 9 females). The mortality of men is $14.4 \%$, and the mortality of women is $9.4 \%$.

In CT imaging with a staging of maximum 4 points and minimum 0 points, the mean value resulted in 1.95. The average radiological stage of the dead patients group was reported as 2.56. There was a correlation between the radiological predictor and the outcome status (p-value: 0.002). In other words, the radiological stage seems statistically significant in predicting death.

127 patients (59.6\%) had chronic disease (hypertension, diabetes mellitus, congestive heart disease, asthma, chronic obstructive pulmonary disease, or other cardiovascular diseases). Of the 26 deceased patients, 13 had a chronic disease, nine had multiple chronic diseases, and four patients did not have any chronic diseases. 
As expected, the effect of additional chronic disease on death was significant (p-value: 0.003464).

The number of smokers was 14 (6.5\%). Of the 26 patients who died, 3 were smokers and 23 were non-smokers. Male patients were more likely to be smokers (p-value: 0.018151 ).

However, there was no significant distribution of smoking regarding age (p-value: 0.2585 ). The effect of smoking on radiological staging could not be demonstrated (p-value: 0.368121 ). We did not see any significant effect on smoking mortality (p-value: 0.2777 ). There was also no significant effect on discharge time (p-value: $0.2496)$.

The average discharge time was 13.8 days. Before starting the study, we expected those with high radiological staging to have a longer discharge time. We strengthened this statistically ( $\mathrm{p}$-value: 0.0049).

In our study, gender and age did not have a significant effect on death (p-values: $0.276459,0.10029)$. Wbc, neutrophil, and magnesium values did not have a significant effect on death (p-values: 0.577682, 0.073708, 0.452292). However, low lymphocyte and high CRP levels showed significant effect on death (p-values: $0.000046,0.010678)$. Low calcium also had a significant effect on death (p-value: 0.000001 ), as did D-dimer and troponin values (p-values: $0.000069,0.003491)$.

\section{Discussion:}

SARS-CoV-2 infection continues to be an urgent public health challenge in China and throughout the world. Most infected patients have mild illness and completely recover after two weeks. However, once infected patients progress to severe illness with acute respiratory distress syndrome, over $10 \%$ of them worsen in a short period of time and $\mathrm{die}^{3}$. As the pathogenesis of SARS-CoV-2 infection is unknown, there is no standard specific treatment and most patients receive symptomatic treatment. Thus, a further understanding of the pathogenesis and treatment of the disease is urgently needed. In Figure 1, we see effects of variable changes on dead and living groups.

Previous studies have found that some routine laboratory biomarkers are out of reference ranges and are higher in more severe disease ${ }^{11}$. In accordance with those studies, we also found that some biomarkers, such as lactate dehydrogenase, troponin and D-dimer, were higher in cases of severe illness, but these biomarkers were not all specific to SARS-CoV-2. Similar to other viral infections, neutrophils showed no obvious change in mild cases, while inflammatory biomarkers such as CRP were increased.

There is a small amount of research that says that smoking could increase the risk of COVID-19 via upregulation of ACE-2 expression, a known cellular entry gateway for SARS-CoV-2 (7,8). However, there are a few inconsistencies with this hypothesis. First of all, the spike protein of the virus is responsible for ACE-2 binding, and it requires its counterpart to be localized on the plasma membrane in order to be subsequently internalized $^{12,13}$. Therefore, the gene expression does not conclusively indicate increased viral infection risk. Second, it is known that ACE-2 expression is downregulated on plasma membranes following SARS-CoV-2 infection because of internalization of ACE-2-virus samples ${ }^{14}$. Third, simple ACE-2 expression on plasma membranes may not be an important element in establishment of a potential risk factor for virus infection. In fact, once the spike protein is bound to ACE-2, the cell is required to trigger a complex series of biochemical activities and molecular signals in order to internalize the virus ${ }^{12}$. The view that overexpression of ACE2 is harmful does not take into account more recent evidence that upregulation of ACE2 may in fact be protective against disease severity ${ }^{9}$. Experimental data suggests that infection with SARS-CoV-2 leads to downregulation of ACE2, and this downregulation is harmful because of uncontrolled ACE and angiotensin II activity ${ }^{8,9}$. It has been observed that decreased ACE2 availability contributes to lung injury and increases the risk of ARDS development ${ }^{9,15}$. Therefore, higher ACE2 expression, while it seems paradoxical, may protect against the acute lung injury caused by COVID-19 ${ }^{16}$.

In our study, we saw smaller-than-expected smoking rates in hospitalized patients who tested positive for COVID-19. That statistic catalyzed this study. We performed our study with inpatients because their progress was easy to follow and similar studies were performed in hospitalized patients. 
14 of the patients in the study were smokers $(6.5 \%)$. In the latest studies on the prevalence of smoking in Turkey, the nationwide rate of smoking was $25.7 \%^{17}$. One in four people in Turkey is a smoker, while in COVID-19 isolation service only a $6.5 \%$ rate of smoking was observed. That supports the theory that smoking hasn't negative impact on COVID-19 development. In the review showing the relationship between smoking and COVID-19 in China, where the smoking prevalence is $26.6 \%, 5960$ patients' smoking rates have changed between 1.4-12.6\%. This study, conducted with patients hospitalized in COVID-19 isolation service, claimed that smoking did not meet the hospitalization criteria for COVID-19, and on the contrary, it may reduce risk. The results of our study are similar to and supportive of these results. At the same time, the CT scores we used to stage the severity of the disease were not statistically related to smoking (p-value: 0.368121 ). We did not see any significant effect of smoking on mortality rate (p-value: 0.2777 ) or on discharge time (p-value: 0.2496$)$.

A semi-quantitative scoring obtained from CT images was used to determine the severity of COVID-19's pulmonary involvement. A CT scan was performed during the first day of the patient's hospitalization. Each lung lobe was scored as $0(0 \%), 1(1-25 \%), 2(26-50 \%), 3(51-75 \%)$, or $4(76-100 \%)$, according to the COVID-19 involvement percentage. The total involvement score of 5 lobes (0-20) was obtained, and a CT evaluation was performed in the lung parenchyma window. Scoring was performed according to the ground glass opacity appearance of COVID-19, which is the typical lung involvement, and according to the percentage of consolidations. In CT imaging with a staging of maximum 4 and minimum 0 points, the mean value was 1.95 .

The average radiological stage was reported as 2.56 in the dead patients' group. There was a correlation between the radiological predictor and the outcome status (p-value: 0.002). It seems that an elevated radiological stage is a predictor of death.

We do not have a standard discharge time at our hospital. When the patients show no fever, no respiratory distress, normalized laboratory tests and good general condition for at least for three days, we discharge them. The average discharge time was 13.8 days. According to our data, we can calculate the average discharge time by scoring their CTs. A high point CT stage will likely delay discharge (p-value: 0.0049).

In conclusion, observation of a consistently low prevalence of smoking among hospitalized COVID-19 cases, together with the potential mechanisms through which nicotine interacts with the inflammatory process and the renin-angiotensin-aldosterone system, highlights that the relationship between smoking and COVID19 should be further investigated. The complex interaction between smoking and the renin-angiotensinaldosterone/ACE-2 systems present multiple challenges to the researcher, the clinician, and the COVID-19 patient. However, CT staging appears to be a very important prognostic factor that may be helpful in the future, especially regarding death and discharge times.

The shortcomings of our study are that our most recent information about current cigarette prevalence in Turkey is from 2014, our patient numbers are low, and we do not have any data on electronic cigarette usage versus traditional heat-based cigarette usage.

\section{References:}

I confirm data availability for this manuscript.

1- $\quad \mathrm{Wu}$ JT, Leung K, Leung GM. Nowcasting and forecasting the potential domestic and international spread of the 2019-nCoV outbreak originating in Wuhan, China: a modelling study. Lancet. 2020;395(10225):689697. doi: 10.1016/S0140-6736(20)30260-9. [PMC free article] [PubMed] [CrossRef] [Google Scholar]

2- $\quad$ Yang X, Yu Y, Xu J, et al. Clinical course and outcomes of critically ill patients with SARS-CoV-2 pneumonia in Wuhan, China: a single-centered, retrospective, observational study. Lancet Respir Med. 2020 doi: 10.1016/s2213-2600(20)30079-5. [PMC free article] [PubMed] [CrossRef] [Google Scholar]

3- Chen N, Zhou M, Dong X, et al. Epidemiological and clinical characteristics of 99 cases of 2019 novel coronavirus pneumonia in Wuhan, China: a descriptive study. Lancet. 2020;395:507-513. [PMC free 
article] [PubMed] [Google Scholar]

4- Chung M, Bernheim A, Mei X, et al. CT imaging features of 2019 novel coronavirus (2019-nCoV). Radiology. 2020;295:202-207. [PMC free article] [PubMed] [Google Scholar]

5- $\quad$ Khot WY, Nadkar MY. The 2019 Novel Coronavirus Outbreak-A Global Threat. J Assoc Physicians India. 2020;68(3):67. [PubMed] [Google Scholar]

6- Eapen M.S., Sharma P., Moodley Y.P., Hansbro P.M., Sohal S.S. Dysfunctional Immunity and Microbial Adhesion Molecules in Smoking-Induced Pneumonia. Am. J. Respir. Crit. Care Med. 2019;199:250-251. doi: 10.1164/rccm.201808-1553LE. [PubMed] [CrossRef] [Google Scholar]

7- Cai G. Bulk and single-cell transcriptomics identify tobacco-use disparity in lung gene expression of ACE2, the receptor of 2019-nCov. medRxiv. 2020:2020.02.05.20020107. doi:10.1101/2020.02.05.20020107 [CrossRef] [Google Scholar]

8- Zhang H., Penninger J.M., Li Y., Zhong N., Slutsky A.S. Angiotensin-converting enzyme 2 (ACE2) as a SARS-CoV-2 receptor: Molecular mechanisms and potential therapeutic target. Intensive Care Med. 2020;46:586-590. doi: 10.1007/s00134-020-05985-9. [PMC free article] [PubMed] [CrossRef] [Google Scholar]

9- Kuba K., Imai Y., Rao S., Gao H., Guo F., Guan B., Huan Y., Yang P., Zhang Y., Deng W., et al. A crucial role of angiotensin converting enzyme 2 (ACE2) in SARS coronavirus-induced lung injury. Nat. Med. 2005;11:875-879. doi: 10.1038/nm1267. [PMC free article] [PubMed] [CrossRef] [Google Scholar]

10- Kunwei Li, Yijie Fang, Wenjuan Li ve ark.,(2020), CT image visual quantitative evaluation and clinical classification of coronavirus disease (COVID-19). Eur Radiol . 2020 Mar 25 : 1-10.doi: 10.1007/s00330-02006817-6

11- Huang C, et al. Clinical features of patients infected with 2019 novel coronavirus in Wuhan, China. Lancet. 2020;395(10223):497-506. doi: 10.1016/S0140-6736(20)30183-5. [PMC free article] [PubMed] [CrossRef] [Google Scholar]

12- Hoffmann M., Kleine-Weber H., Schroeder S., Kruger N., Herrler T., Erichsen S., Schiergens T.S., Herrler G., Wu N.H., Nitsche A., et al. SARS-CoV-2 cell entry depends on ACE2 and TMPRSS2 and is blocked by a clinically proven protease inhibitor. Cell. 2020 doi: 10.1016/j.cell.2020.02.052. [PMC free article] [PubMed] [CrossRef] [Google Scholar]

13- Jia H.P., Look D.C., Shi L., Hickey M., Pewe L., Netland J., Farzan M., Wohlford-Lenane C., Perlman S., McCray P.B., Jr. ACE2 receptor expression and severe acute respiratory syndrome coronavirus infection depend on differentiation of human airway epithelia. J. Virol. 2005;79:14614-14621. doi: 10.1128/JVI.79.23.14614-14621.2005. [PMC free article] [PubMed] [CrossRef] [Google Scholar]

14- Glowacka I., Bertram S., Herzog P., Pfefferle S., Steffen I., Muench M.O., Simmons G., Hofmann H., Kuri T., Weber F., et al. Differential downregulation of ACE2 by the spike proteins of severe acute respiratory syndrome coronavirus and human coronavirus NL63. J. Virol. 2010;84:1198-1205. doi: 10.1128/JVI.0124809. [PMC free article] [PubMed] [CrossRef] [Google Scholar]

15- Dijkman R., Jebbink M.F., Deijs M., Milewska A., Pyrc K., Buelow E., van der Bijl A., van der Hoek L. Replication-dependent downregulation of cellular angiotensin-converting enzyme 2 protein expression by human coronavirus NL63. J. Gen. Virol. 2012;93:1924-1929. doi: 10.1099/vir.0.043919-0. [PubMed] [CrossRef] [Google Scholar]

16- Gurwitz D. Angiotensin receptor blockers as tentative SARS-CoV-2 therapeutics. Drug Dev. Res. 2020 doi: 10.1002/ddr.21656. [PMC free article] [PubMed] [CrossRef] [Google Scholar]

17- Deliana Kostova, Linda Andes. Cigarette Prices and Smoking Prevalence After a Tobacco Tax Increase - Turkey, 2008 and 2012 
Figure-1: Box plots of alive and deceased groups for different variables

Figure-1: Box plots between alive and deceased groups for different variables
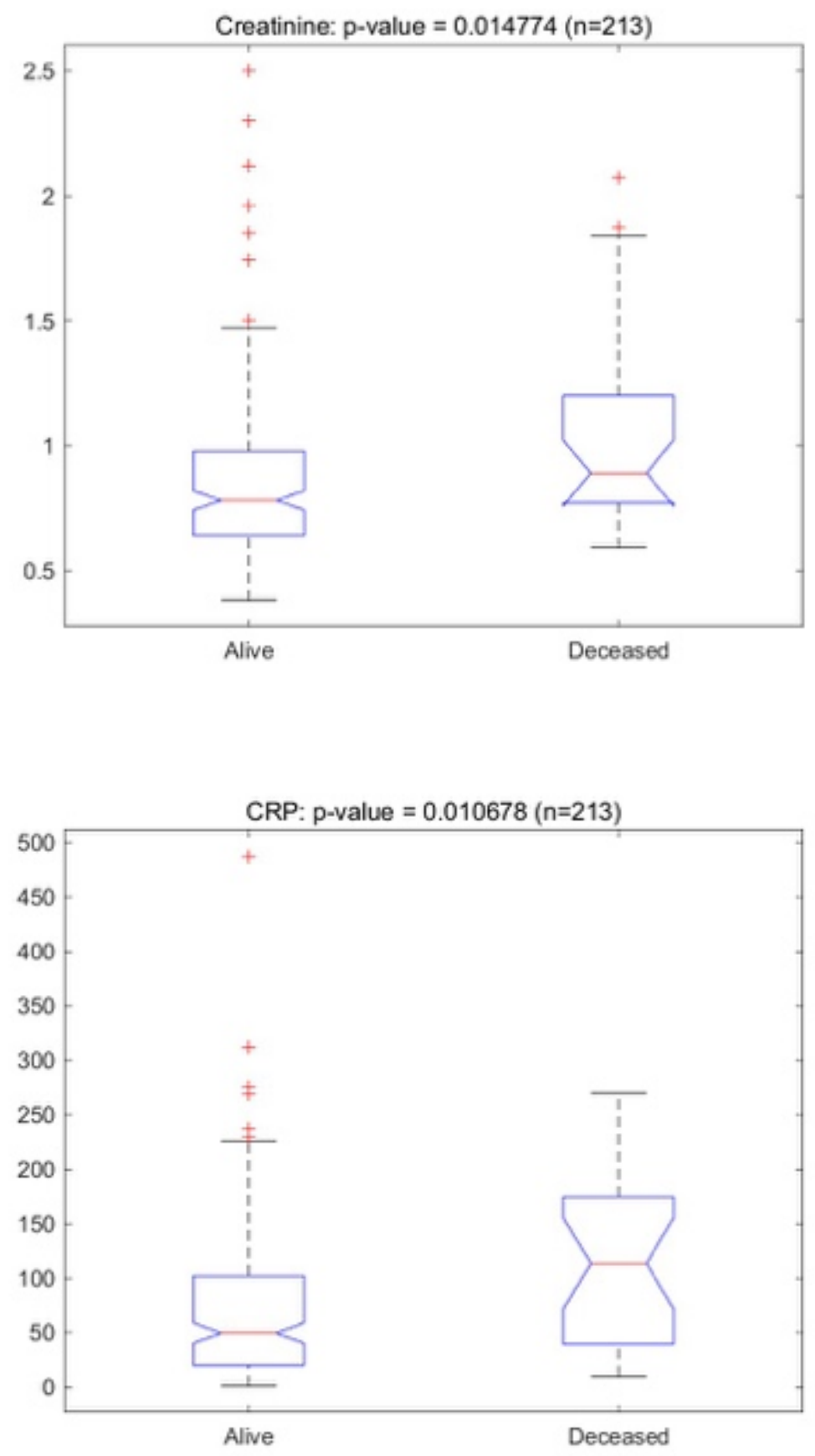

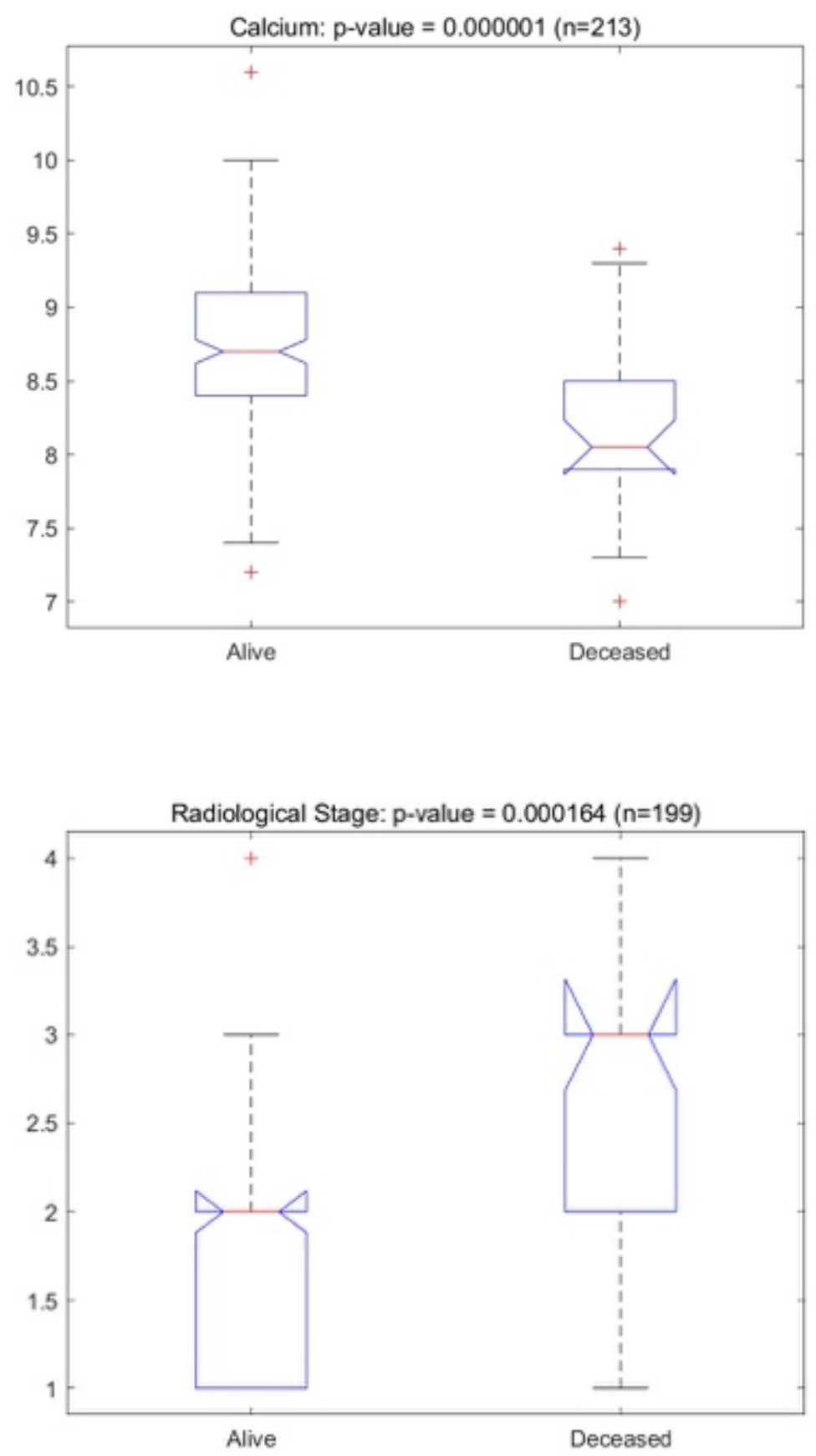
Platelets: $p$-value $=0.036307(n=213)$

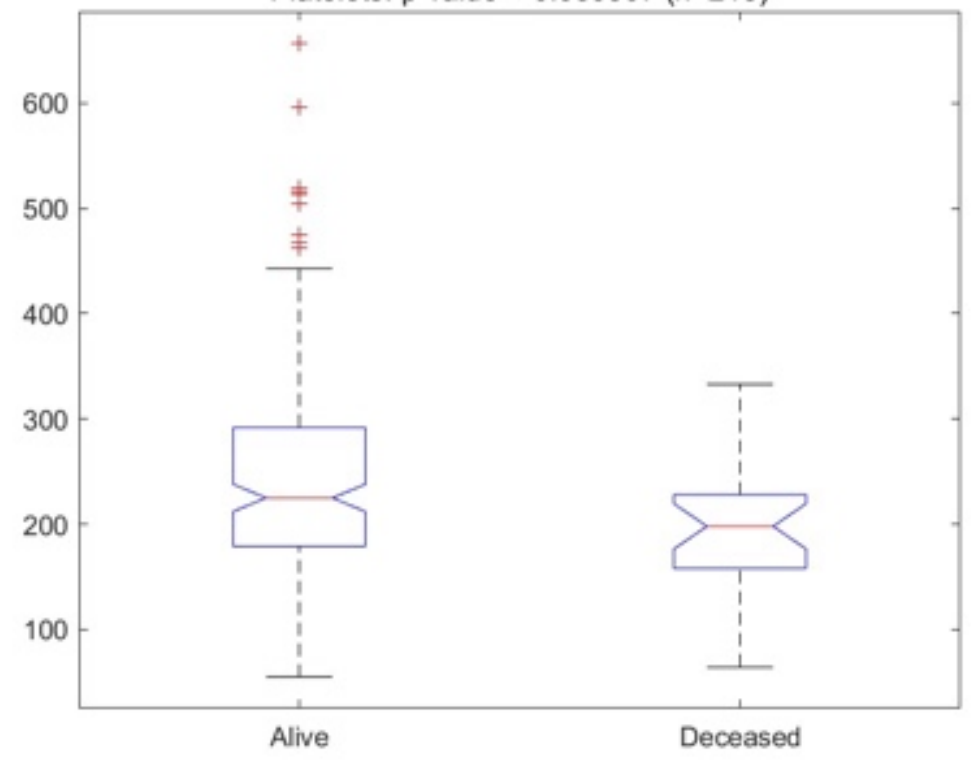

Lymphocyte: $p$-value $=0.000046(n=213)$

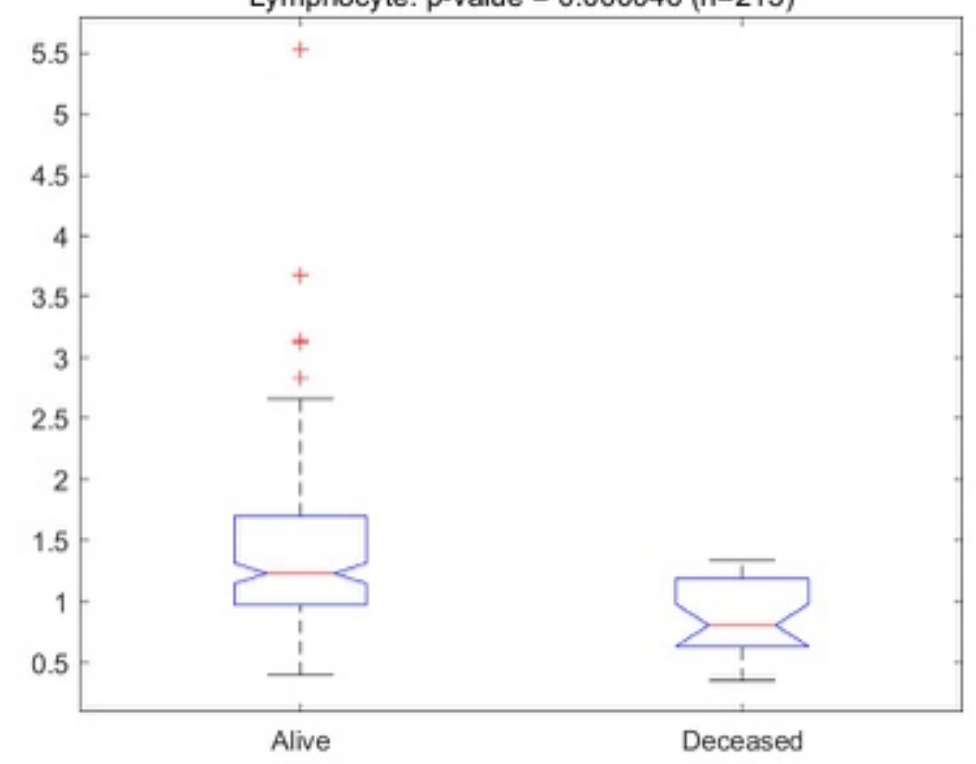



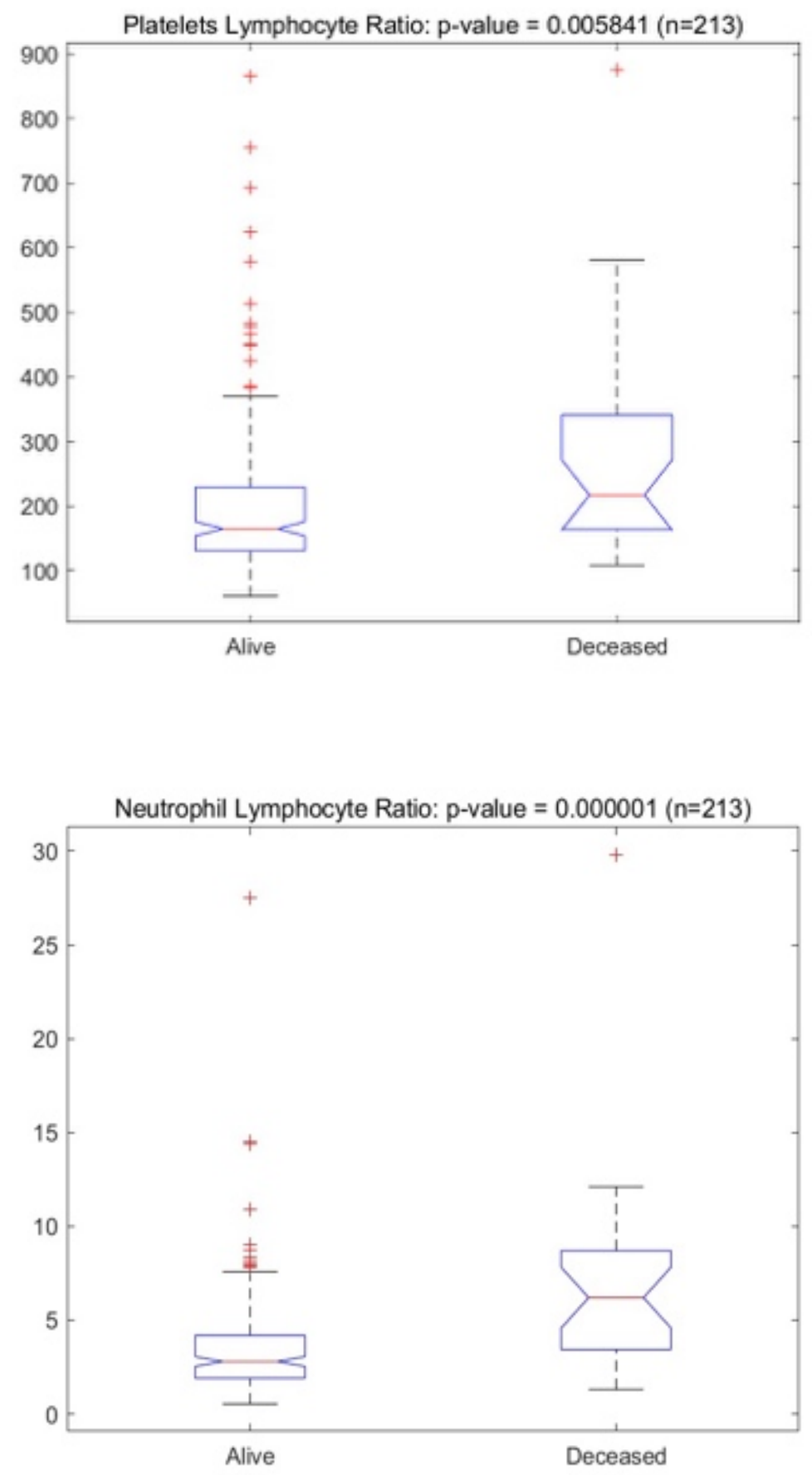

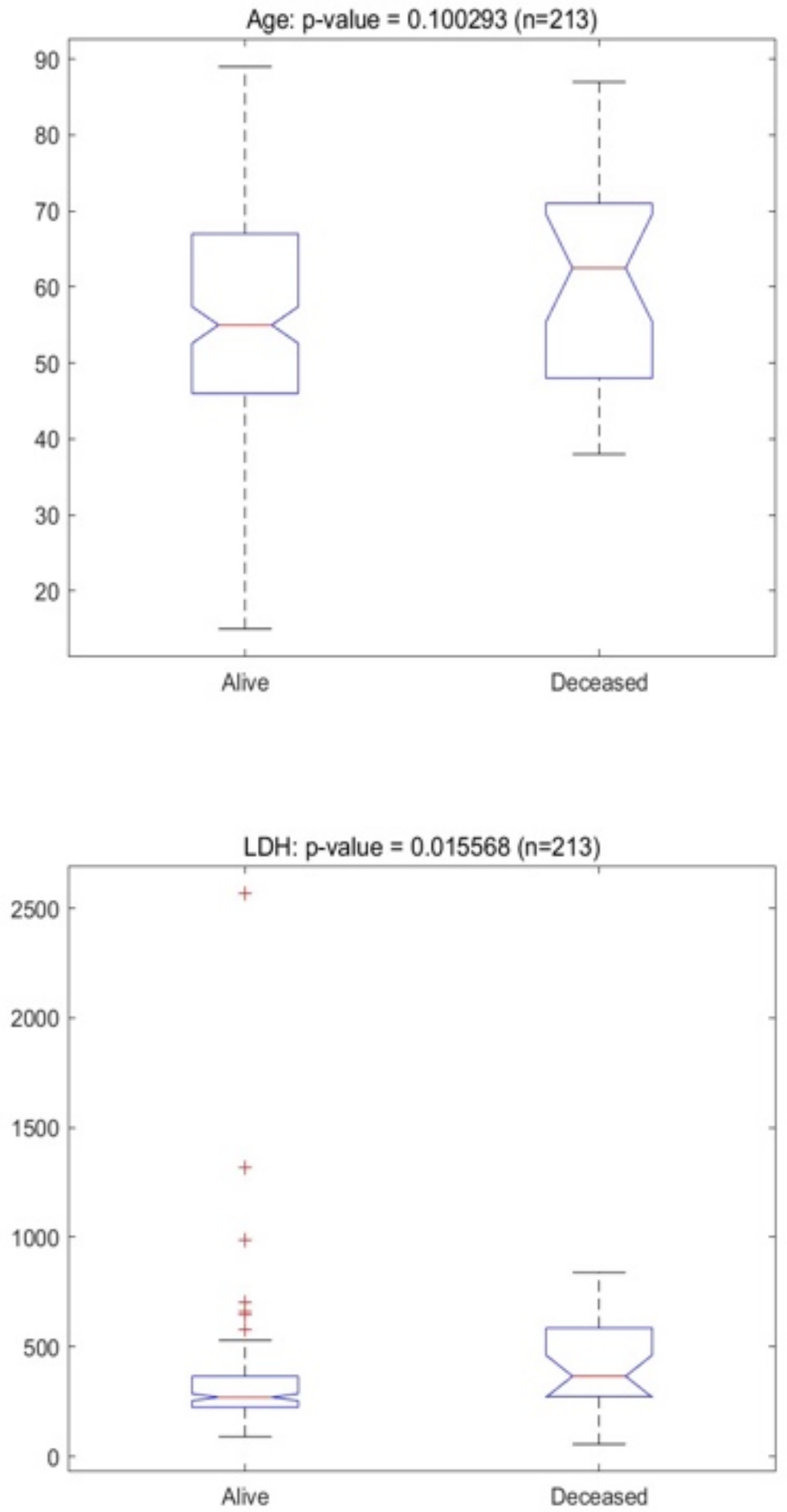

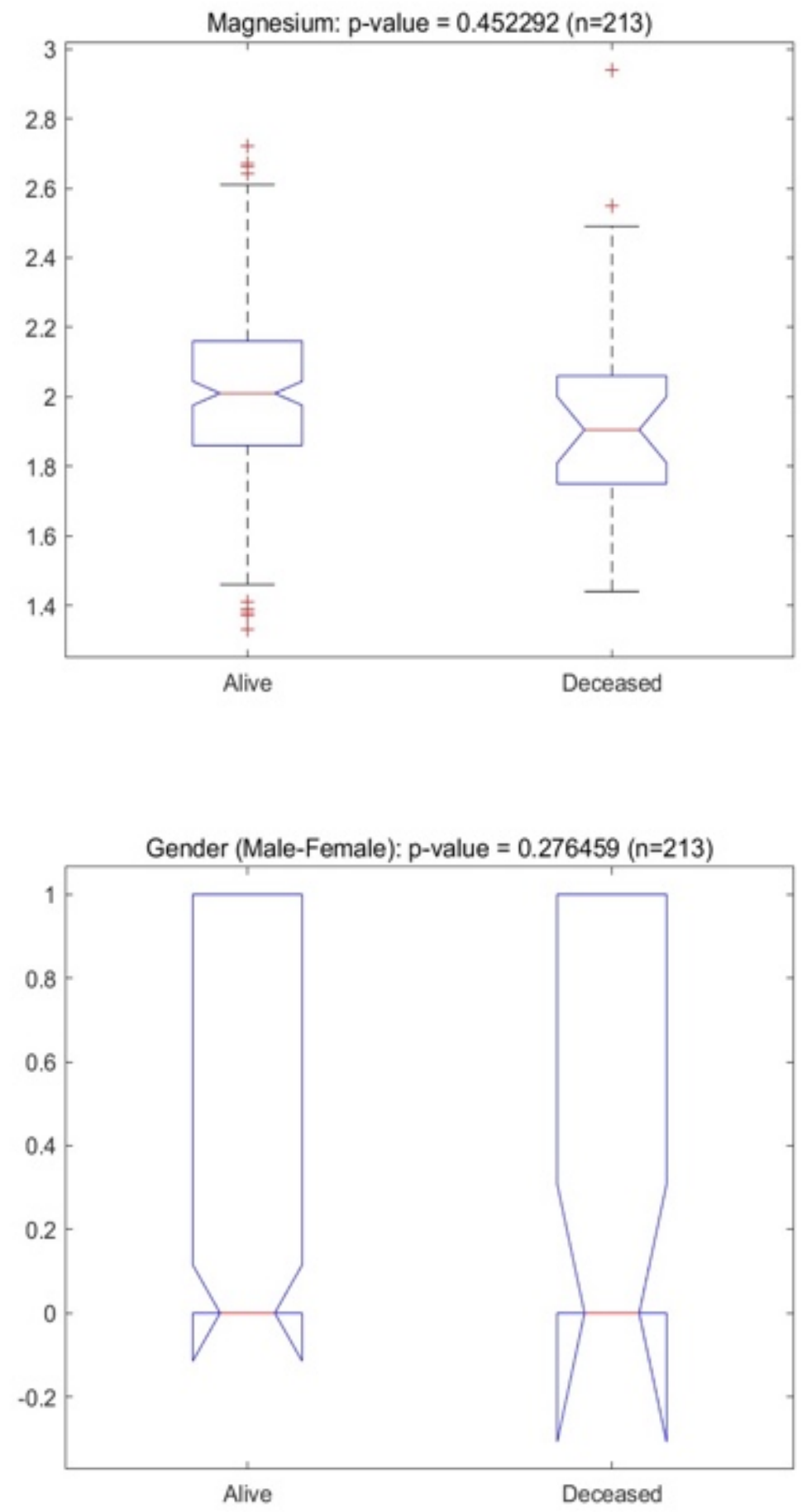

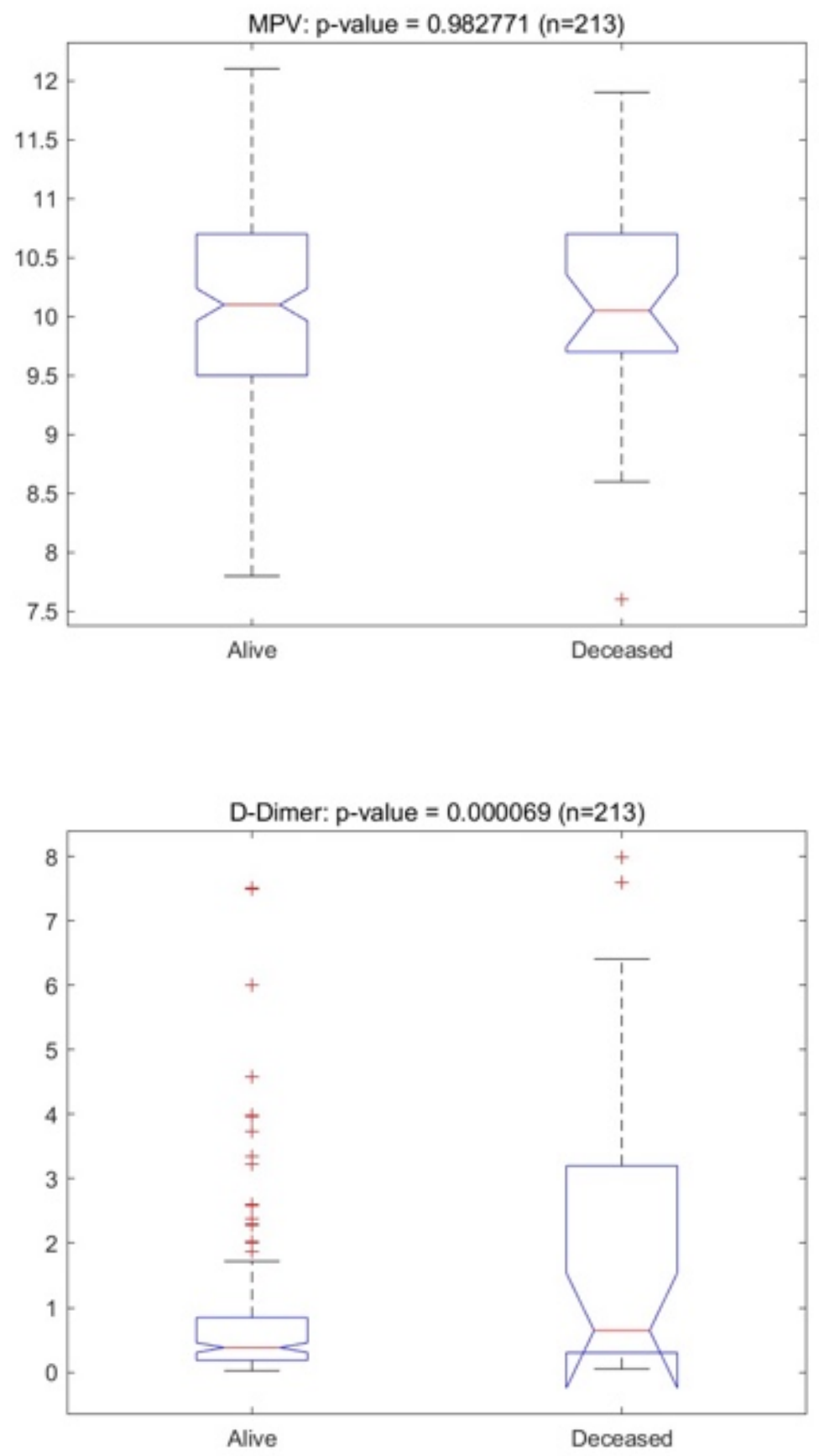

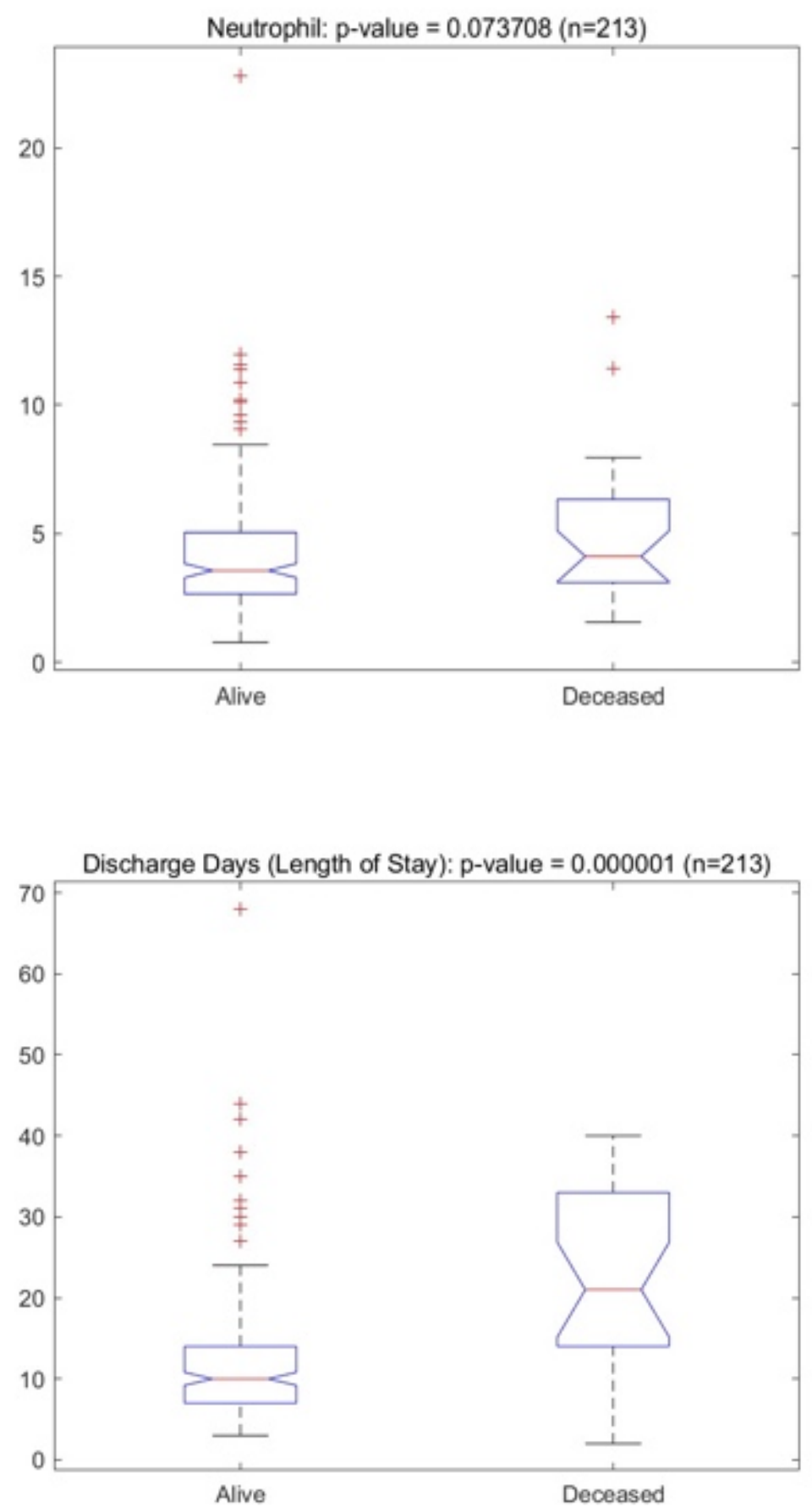
Number of Additional Conditions: $p$-value $=0.003464(n=213)$

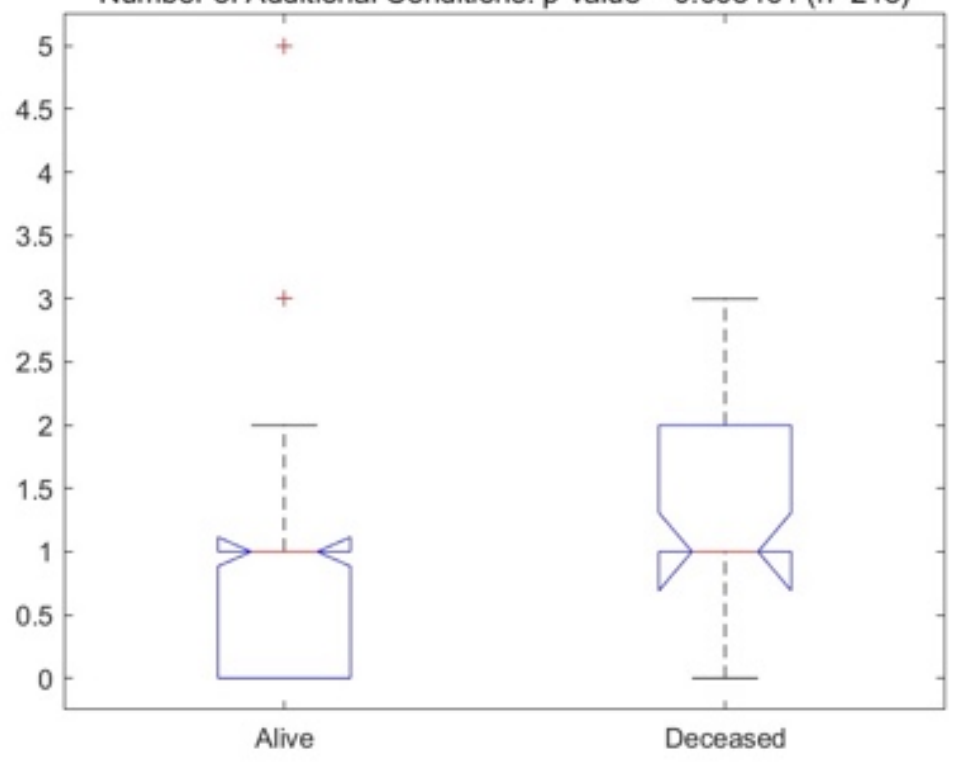

Ferritin: $p$-value $=0.103135(n=213)$

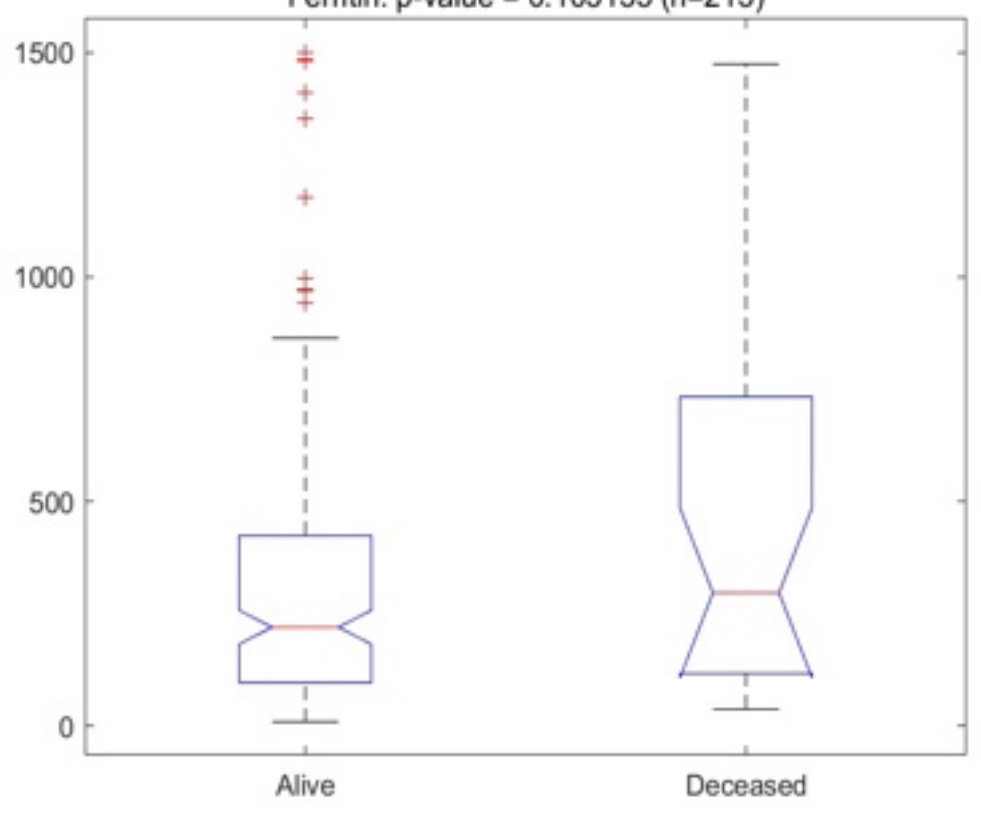



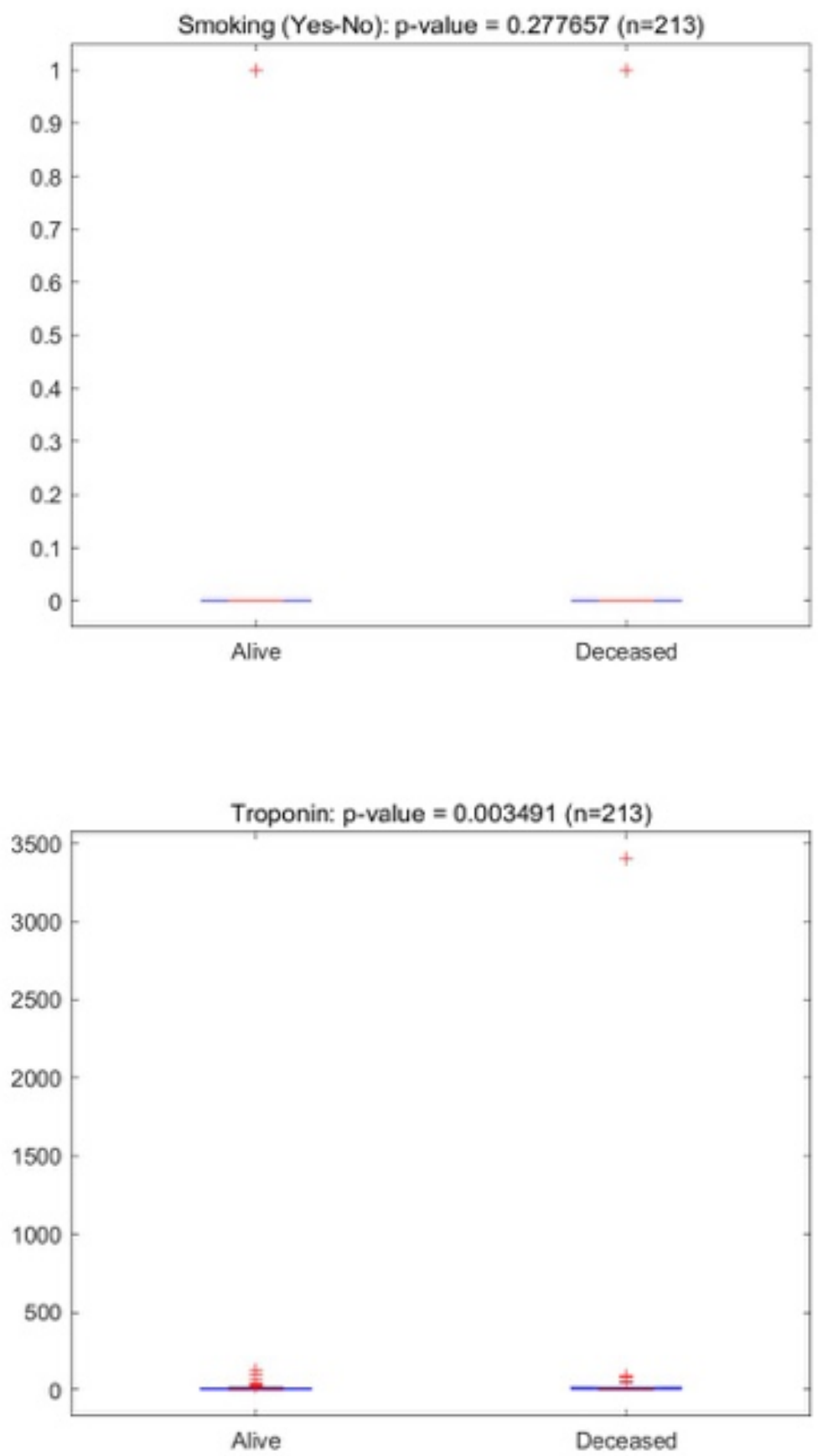